\title{
Correlations Without Synchrony
}

\author{
Carlos D. Brody* \\ Computation and Neural Systems Program, California Institute of Technology, Pasadena, \\ CA 91125, U.S.A.
}

Peaks in spike train correlograms are usually taken as indicative of spike timing synchronization between neurons. Strictly speaking, however, a peak merely indicates that the two spike trains were not independent. Two biologically plausible ways of departing from independence that are capable of generating peaks very similar to spike timing peaks are described here: covariations over trials in response latency and covariations over trials in neuronal excitability. Since peaks due to these interactions can be similar to spike timing peaks, interpreting a correlogram may be a problem with ambiguous solutions. What peak shapes do latency or excitability interactions generate? When are they similar to spike timing peaks? When can they be ruled out from having caused an observed correlogram peak? These are the questions addressed here. The previous article in this issue proposes quantitative methods to tell cases apart when latency or excitability covariations cannot be ruled out.

\section{Introduction}

Suppose that the spike trains of two neurons, recorded simultaneously during many identically prepared experimental trials, have been obtained. A standard method to assess the presence of interactions between the spike trains-beyond those expected by chance given each neuron's peristimulus time histogram (PSTH) - is to compute their shuffle-corrected crosscorrelogram Perkel, Gerstein, \& Moore, 1967; Palm, Aertsen, \& Gerstein, 1988; Aertsen, Gerstein, Habib, \& Palm, 1989. The name shuffle-corrected cross-correlogram will be henceforth abbreviated to cross-covariogram or simply covariogram. ${ }^{1}$ Peaks in covariograms are usually interpreted as signaling the presence of spike timing synchronization between the two neurons. Strictly speaking, however, a peak in a covariogram merely indicates that the two spike trains were not independent, and synchronizing the spike

\footnotetext{
* Present address: Instituto de Fisiología Celular, UNAM, México D. F. 04510, México.

1 The abbreviation covariogram comes from the fact that the computation of the shufflecorrected cross-correlogram is exactly analogous to the computation of covariance when the variables of interest are scalars rather than spike trains (Aertsen et al., 1989; Brody, 1997a).
}

Neural Computation 11, 1537-1551 (1999) C 1999 Massachusetts Institute of Technology 
times of the two neurons is only one of many possible ways to depart from independence. Figure 1 shows three very different ways to depart from independence, all of which lead to similar covariograms. Despite their similarity, each case should be interpreted very differently, in terms of both the mechanisms that could cause it and its functional significance. All three types of covariations illustrated (which will be called spike timing, or latency, or excitability covariations) are biologically plausible. Thus, being aware of the different possibilities, and disambiguating them, is important.

This article will explain how latency and excitability covariations lead to a peak in the covariogram (spike timing covariations have been treated before, e.g. Perkel et al., 1967). The article will also explain under what conditions their peaks are similar to peaks caused by spike timing covariations. Rules of thumb for being alert to the possibility of ambiguous covariograms will be emphasized; the previous article in this issue describes more quantitative methods, which attempt to dispel the ambiguity when it arises. A preliminary version of the results presented here has appeared in abstract form (Brody, 1997b).

\section{Notation and Correlogram Methods}

The spike trains of two cells will be represented by two time-dependent functions, $S_{1}(t)$ and $S_{2}(t)$. They will be assumed binned and collected over many identically prepared experimental trials, indexed by a superscript $r$. For times outside the $r$ th trial, $S_{1}^{r}(t)$ will be defined to be zero, and similarly for $S_{2}^{r}(t)$. The cross-correlogram of each trial is then

$$
C^{r}(\tau) \equiv \sum_{t=-\infty}^{\infty} S_{1}^{r}(t) S_{2}^{r}(t+\tau) \equiv S_{1}^{r} \odot S_{2}^{r}
$$

Let \langle\rangle represent averaging over trials $r$, and define $P_{i}(t) \equiv\left\langle S_{i}^{r}(t)\right\rangle$. If spike times are measured relative to a stimulus, this is the PSTH of $S_{i}$. The covariogram of $S_{1}$ and $S_{2}$ is then defined as

$$
\begin{aligned}
V & \equiv\left\langle\left(S_{1}^{r}-P_{1}\right) \odot\left(S_{2}^{r}-P_{2}\right)\right\rangle \\
& =\left\langle S_{1}^{r} \odot S_{2}^{r}\right\rangle-P_{1} \odot P_{2} .
\end{aligned}
$$

The two terms in equation 2.2 are known as the raw cross-correlogram $R=$ $\left\langle S_{1}^{r} \odot S_{2}^{r}\right\rangle$ and the shuffle corrector ${ }^{2} K=P_{1} \odot P_{2}$. If $S_{1}$ and $S_{2}$ are independent,

\footnotetext{
2 The shift predictor (Perkel et al., 1967) is very similar to the shuffle corrector, except that $K$ is replaced by $D=\left\langle S_{1}^{r} \odot S_{2}^{\Pi(r)}\right\rangle$, where $\Pi(r)$ is some permutation of the stimulus presentations $r$ (and the corresponding substitution is made in equation 2.2). If different trials are independent of one another, then the expected value of the shift predictor $D$ is equal to the expected value of the shuffle corrector $K$. Thus, they are both estimators of the same function. In practice, it is preferable to use $K$ instead of $D$ since the former is a less noisy estimator: $K$ can be written as the average of $D$, taken over the set of all possible permutations $\Pi$ (Palm et al., 1988).
} 
then the expected value of $V$ is zero:

$$
\begin{aligned}
E\{V\} & =E\left\{\left(S_{1}^{r}-P_{1}\right) \odot\left(S_{2}^{r}-P_{2}\right)\right\} \\
& =E\left\{S_{1}^{r}-P_{1}\right\} \odot E\left\{S_{2}^{r}-P_{2}\right\}=0 .
\end{aligned}
$$

Therefore, significant departures of $V$ from zero indicate that the two cells were not independent, regardless of what distributions that $S_{1}^{r}$ and $S_{2}^{r}$ were drawn from. Estimating the significance of departures of $V$ from 0 requires some assumptions. For the null hypothesis, it will be assumed that $S_{1}$ is independent of $S_{2}$, different trials of $S_{1}$ are independent of each other, and different bins within each trial of $S_{1}$ are independent of each other (similar assumptions for the trials and bins of $S_{2}$ will also be made). If $P_{i}(t)$ and $\sigma_{i}^{2}(t)$ are the mean and variance of $S_{i}^{r}(t)$ over trials $r$ and $N_{\text {trials }}$ is the number of

\section{A: Spike timing}
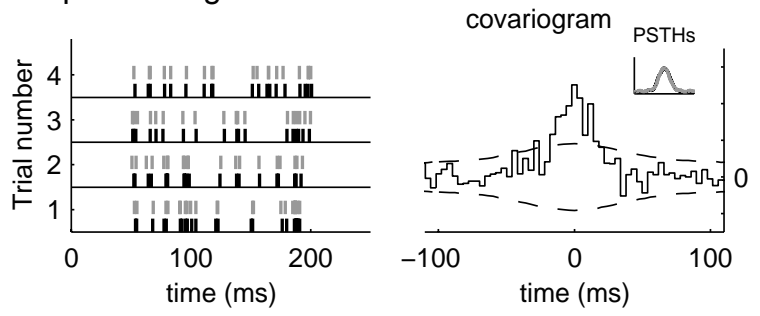

\section{B: Latency}
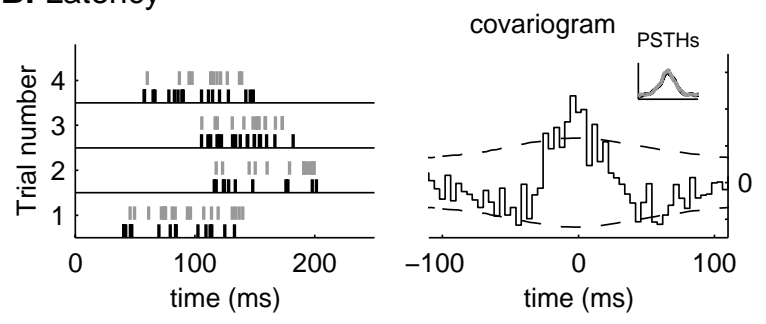

\section{C: Excitability}
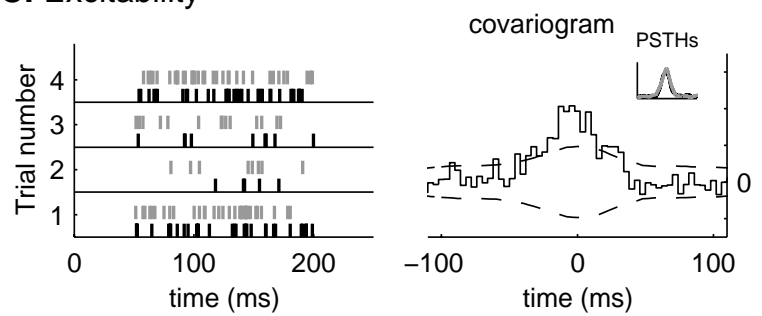
trials in the experiment, then the variance in the null hypothesis for $V$ is

$$
\sigma_{V}^{2}(t)=\left(\sigma_{1}^{2} \odot \sigma_{2}^{2}+P_{1}^{2} \odot \sigma_{2}^{2}+\sigma_{1}^{2} \odot P_{2}^{2}\right) / N_{\text {trials }} .
$$

In practice, one uses the sample means and variances to calculate $\sigma_{V}(t)$; the $2 \sigma$ limits, calculated in this way, are displayed as dashed lines in the covariograms throughout this article. ${ }^{3}$ While $\sigma$ is a general measure of the spread of a distribution, more assumptions must be made in order to use it to assign a specific number to a significance limit; for example, if the distribution is assumed gaussian, then $2 \sigma$ represents the $95 \%$ confidence limit. No particular assumption will be made here.

Joint peristimulus time histograms, (JPSTHs) (Aertsen et al., 1989) will also be used. The unnormalized JPSTH is a matrix of covariances with elements defined as

$$
J\left(t_{1}, t_{2}\right)=\left\langle S_{1}^{r}\left(t_{1}\right) S_{2}^{r}\left(t_{2}\right)\right\rangle-\left\langle S_{1}^{r}\left(t_{1}\right)\right\rangle\left\langle S_{2}^{r}\left(t_{2}\right)\right\rangle,
$$

Figure 1: Facing page. Three types of covariations. Despite being very different, all three shuffle-corrected correlograms (henceforth called covariograms) look very similar. Each row illustrates a type of covariation: On the left is a raster plot of two simulated cells, and on the right is the covariogram of spike trains made in a similiar fashion. (Parameters used for rasters on the left were set to extreme values to emphasize illustrative clarity; parameters used for covariograms on the right were set to physiologically plausible values.) (A) On each trial, most spikes in cell 1 have a corresponding, closely timed spike in cell 2 . Both cells have the same response latency and overall firing rate in all trials. This will be called a spike timing covariation. (B) Spikes in cell 1 do not have a corresponding spike in cell 2, on each trial, the two spike trains were generated independently of each other. But the overall latency of the response varies together over trials. (The word latency will be used here to indicate the time shift of the whole response, not just of the first spike.) (C) On each trial, the spikes for the two cells were generated independently of each other, but the total magnitude of the response (which will be called the excitability) varies together over trials. Zero counts on the covariogram $y$ axes is the expected value if the two cells are independent; the dashed lines are significance limits. The inset at the top right of each covariogram shows the PSTHs of the two cells involved, plotted on axes that are $250 \mathrm{~ms}$ wide and $60 \mathrm{~Hz}$ tall.

\footnotetext{
${ }^{3}$ To see where equation 2.4 comes from, consider two independent scalars $x$ and $y$ with means $p_{x}$ and $p_{y}$ and variances $\sigma_{x}^{2}$ and $\sigma_{y}^{2}$, respectively. The variance of their product is $E\left\{x^{2}\right\} E\left\{y^{2}\right\}-E\{x\}^{2} E\{y\}^{2}=\left(\sigma_{x}^{2}+p_{x}^{2}\right)\left(\sigma_{y}^{2}+p_{y}^{2}\right)-p_{x}^{2} p_{y}^{2}=\sigma_{x}^{2} \sigma_{y}^{2}+p_{x}^{2} \sigma_{y}^{2}+\sigma_{x}^{2} p_{y}^{2}$. Equation 2.4 is analogous. The factor of $N_{\text {trials }}$ comes from averaging over trials.
} 
while the normalized JPSTH is a matrix of correlation coefficients with elements defined as

$$
J_{N}\left(t_{1}, t_{2}\right)=\frac{J\left(t_{1}, t_{2}\right)}{\sigma_{1}\left(t_{1}\right) \sigma_{2}\left(t_{2}\right)},
$$

If $S_{1}\left(t_{1}\right)$ and $S_{2}\left(t_{2}\right)$ are independent, then the expected values of $J\left(t_{1}, t_{2}\right)$ and $J_{N}\left(t_{1}, t_{2}\right)$ are zero. Correlation coefficients are bounded within $[-1,1]$. If $J_{N}\left(t_{1}, t_{2}\right)=1$, then $S_{1}\left(t_{1}\right)$ and $S_{2}\left(t_{2}\right)$ are perfectly correlated (that is, $S_{1}\left(t_{1}\right)=$ $\alpha S_{2}\left(t_{2}\right)$ for some positive constant $\alpha$ ), while if $J_{N}\left(t_{1}, t_{2}\right)=-1$, then $S_{1}\left(t_{1}\right)$ and $S_{2}\left(t_{2}\right)$ are perfectly anticorrelated (that is, $\left.S_{1}\left(t_{1}\right)=-\alpha S_{2}\left(t_{2}\right)\right)$. The JPSTHs displayed in the figures here are all normalized JPSTHs.

The covariogram $V$ can be obtained from the unnormalized JPSTH $J$ by summing along $t_{1}$ while keeping $\tau=t_{2}-t_{1}$ constant.

\section{What Covariogram Shapes Do Latency and Excitability Covariations Generate?}

3.1 Latency Covariations. Consider the responses of two independent neurons. Since they are independent, their covariogram is zero (within sampling noise); hence, the raw cross-correlogram and the shuffle corrector are approximately equal:

$$
\underbrace{V}_{\text {covariogram }}=\underbrace{R}_{\text {raw } x \text {-corrector }}-\underbrace{K}_{\text {corrector }} \approx 0 \Longrightarrow K \approx R .
$$

Now for each trial $r$, take the responses of both neurons and shift both of their spike trains, together by some amount of time $t^{r}$ (the shift time $t^{r}$ should be different for different trials). This type of interaction between the neurons is dubbed here as a latency covariation. How will it affect $V$ ?

Let us ask how it affects each of the two terms of $V-R$ and $K$. The raw correlogram $R$ will not be affected, since it depends on only relative spike times between the two neurons (see equation 2.1), and on each trial both spike trains were shifted together. In contrast, the shuffle corrector $K$ will be affected. It is the correlogram of the two PSTHs, and the PSTHs are broadened by the temporal jitter introduced by the shifts $t^{r}$. Thus $K$ is broader than before the latency shifts. Since the total number of spikes remains the same, the integral of the PSTHs will not have changed; nor will the integral of $K$ have changed. In summary, the latency shifts will make $K$ broader, and therefore shallower, while having no effect on $R$. Figure 2 shows a schematic of how subtracting the broadened, shallowed $K$ from $R$ leaves a peak in $R$ outstanding in $V$. The peak is flanked by slight negative dips. The most important point to notice about this schematic is that the width and shape of the peak in $V$ are largely determined by the width and shape of the peak in $R$. 
Unless the latency shifts are very large, the width of the peak in $R$, and hence in $V$, will be smaller but of the same order of magnitude as the width of the peak in $K$, which in turn is determined by the width of peaks in the cell's PSTHs. Figures 2B-2F show a numerical experiment illustrating latency covariations. The covariogram peak width is $\approx 50 \mathrm{~ms}$, while PSTH peak widths are $\approx 100 \mathrm{~ms}$. For the simple Poisson-like processes used here and for symmetrical cells, the autocovariograms of each cell (see Figures $2 \mathrm{E}$ and $2 \mathrm{~F}$ ) have a shape similar to the crosscovariogram of the two cells (see Figure 2C).

3.2 Excitability Covariations. Consider a cell whose response can be characterized as the sum of a stimulus-induced response plus a background

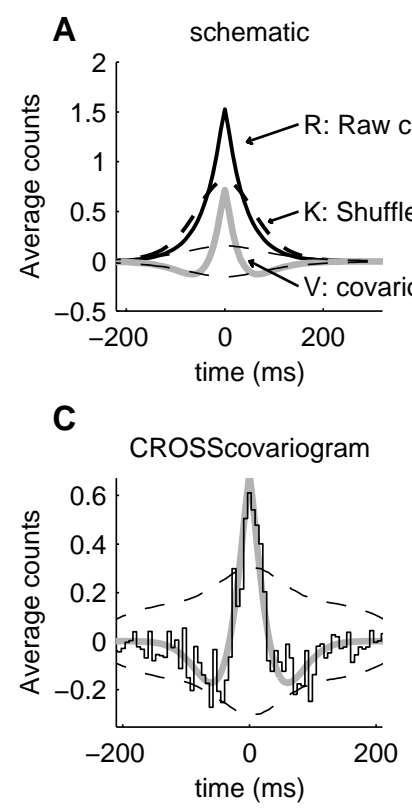

B rasters
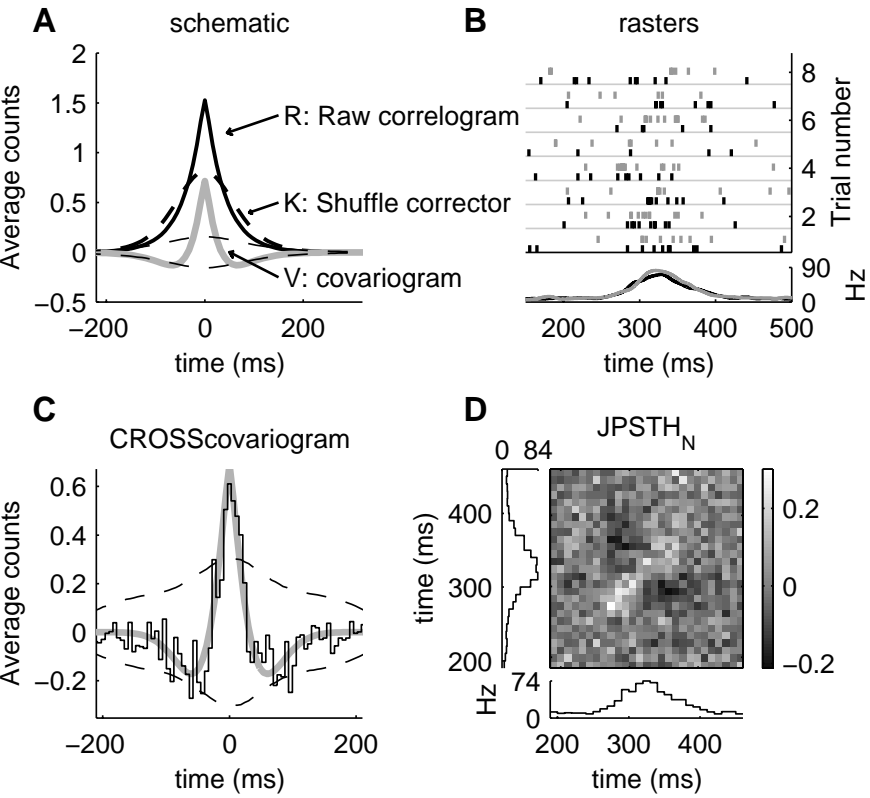

E AUTOcovariogram, cell 1

F AUTOcovariogram, cell 2
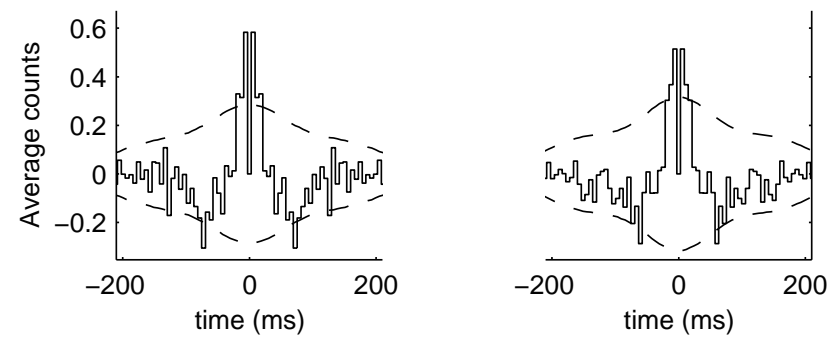
firing rate. Let us write this in terms of firing rates $^{4}$ as

$\underbrace{F^{r}(t)}_{\text {Firing rate during trial } r}=\underbrace{\zeta^{r} Z(t)}_{\text {Stimulus induced }}+\underbrace{\beta^{r} B}_{\text {Background }}$.

Here $Z(t)$ is the typical stimulus-induced firing rate, $B$ is a constant function over the time of a trial, representing the typical background firing rate, and two "gain" factors, $\zeta^{r}$ and $\beta^{r}$, have been included to represent possible changes in the state of the cell (e.g., changes over trials in the resting potential of the cell Carandini \& Ferster, 1997). The gain factors $\zeta^{r}$ and $\beta^{r}$ will be allowed to be different for different trials. Two assumptions are being made here: (1) state changes are slower than the time of a single trial, and (2) the greatest effect of state changes is on the magnitude of the background and stimulus-induced rates, not on their temporal shape. These assumptions allow factoring out the effect of state changes into the scalar gain factors $\zeta^{r}$ and $\beta^{r}$.

Figure 2: Facing page. Latency covariations. (A) Schematic of how latency covariations lead to a peaked covariogram (see the text for explanation). (B) Eight out of 200 artificial rasters used to illustrate latency covariations. Below the rasters are the smoothed PSTHs of both cells. The spike trains were made by simulating two independent Poisson cells, each raster pair of which was then shifted together in time by a random amount drawn anew for each trial from a gaussian distribution with mean $0 \mathrm{~ms}$ and standard deviation $15 \mathrm{~ms}$. The time-varying firing rate from which Poisson events for each cell were generated, before the time shifts, was $(100 \mathrm{~Hz}) \exp \left((t-100)^{2} /\left(2 \cdot 40^{2}\right)\right)$ for $t>100$, zero otherwise, with $t$ in milliseconds. After the time shifts, independent events at a rate of 10 $\mathrm{Hz}$ were added to each cell to represent background firing. (C) Covariogram of the two cells. The thick gray line is the analytical expected value of the covariogram, computed with knowledge of the parameters and procedures used to generate the spike trains. Dashed lines are significance limits. (D) Normalized JPSTH of the two cells, flanked by their PSTHs. Notice the diagonal peak and weak but present off-diagonal troughs. (E, F) Autocovariograms of each of the two cells. These are computed following equation 2.2, except one cell is used, not two, and the central bin $(\tau=0)$, which for autocovariograms is much larger than any other, has been arbitrarily set to zero here for display purposes.

\footnotetext{
${ }^{4}$ The description given in equation 3.2 amounts to describing the cell with a generative model, but all that is being specified about the model is the expected value of its response on each trial. Thus, if $M^{r}(t)$ is the model's response during trial $r$, then $F^{r}(t)=E\left\{M^{r}(t)\right\}$. Note that the expectation here is not taken across trials but is the expected response for a single trial. Think of this as fixing the model's parameters at values appropriate for trial $r$ and averaging over many runs of the model at those parameters.
} 
Now take two cells, indexed by the subscripts 1 and 2, with responses characterized as in equation 3.2. Suppose their only interaction is through their gain parameters. This has been dubbed here an excitability covariation. What is their covariogram $V$ ? In what follows, write the covariance of two scalars $a$ and $b$ as $\operatorname{cov}(a, b) \equiv\langle a b\rangle-\langle a\rangle\langle b\rangle$, and drop the superscripts $r$ for legibility. Using equations 2.2 and 3.2 and the fact that the gain parameters factor out,

$$
\begin{aligned}
V= & \overbrace{\operatorname{cov}\left(\zeta_{1}, \zeta_{2}\right)}^{\text {amplitude }} \overbrace{Z_{1} \odot Z_{2}}^{\text {shape }}+\operatorname{cov}\left(\zeta_{1}, \beta_{2}\right) Z_{1} \odot B_{2} \\
& +\operatorname{cov}\left(\beta_{1}, \zeta_{2}\right) B_{1} \odot Z_{2}+\operatorname{cov}\left(\beta_{1}, \beta_{2}\right) B_{1} \odot B_{2} .
\end{aligned}
$$

Similarly, the JPSTH (before normalization) is

$$
\begin{aligned}
J\left(t_{1}, t_{2}\right)= & \operatorname{cov}\left(\zeta_{1}, \zeta_{2}\right) Z_{1}\left(t_{1}\right) Z_{2}\left(t_{2}\right)+\operatorname{cov}\left(\zeta_{1}, \beta_{2}\right) Z_{1}\left(t_{1}\right) B_{2} \\
& +\operatorname{cov}\left(\beta_{1}, \zeta_{2}\right) B_{1} Z_{2}\left(t_{2}\right)+\operatorname{cov}\left(\beta_{1}, \beta_{2}\right) B_{1} B_{2},
\end{aligned}
$$

where the time dependence of $B_{1}$ and $B_{2}$ has been dropped from the notation since they are constant functions.

When the stimulus-induced firing rate is much greater than the background firing rate, the first term in equation 3.3 is the dominant one. The shape of $V$ will then be given by $Z_{1} \odot Z_{2}$ (in this limiting case, this is also the shape of the corrector $K$, which has a width determined by the width of peaks in the cell's PSTHs), while the amplitude of $V$ will be given by $\operatorname{cov}\left(\zeta_{1}, \zeta_{2}\right)$. A similar point has been made by Friston (1995), whose work is discussed in the companion article in this issue; (see also Vaadia, Aertsen, \& Nelken, 1995). Figure 3 shows a numerical experiment illustrating excitability covariations. For the simple Poisson-like processes used here and for symmetrical cells, the autocovariograms of the cells (panel D) are similar to the cross-covariogram (panel B), much as was the case with latency covariations (see Figure 2).

An easily computable and telltale measure of excitability covariations is the integral (i.e., sum) of the covariogram, since it is proportional to the covariation in the mean firing rates of the two cells: $\sum_{\tau} V(\tau)=\operatorname{cov}\left(n_{1}^{r}, n_{2}^{r}\right)$, where $n_{i}^{r}$ is the total number of spikes fired by cell $i$ during trial $r$. For completeness, the proof follows:

$$
\begin{aligned}
\sum_{\tau=-\infty}^{\infty} C^{r}(\tau) & =\sum_{\tau=-\infty}^{\infty} \sum_{t=-\infty}^{\infty} S_{1}^{r}(t) S_{2}^{r}(t+\tau)=\sum_{p, q} S_{1}^{r}(p) S_{2}^{r}(q) \\
& =\sum_{p} S_{1}^{r}(p) \sum_{q} S_{2}^{r}(q) \\
& =n_{1}^{r} n_{2}^{r} .
\end{aligned}
$$



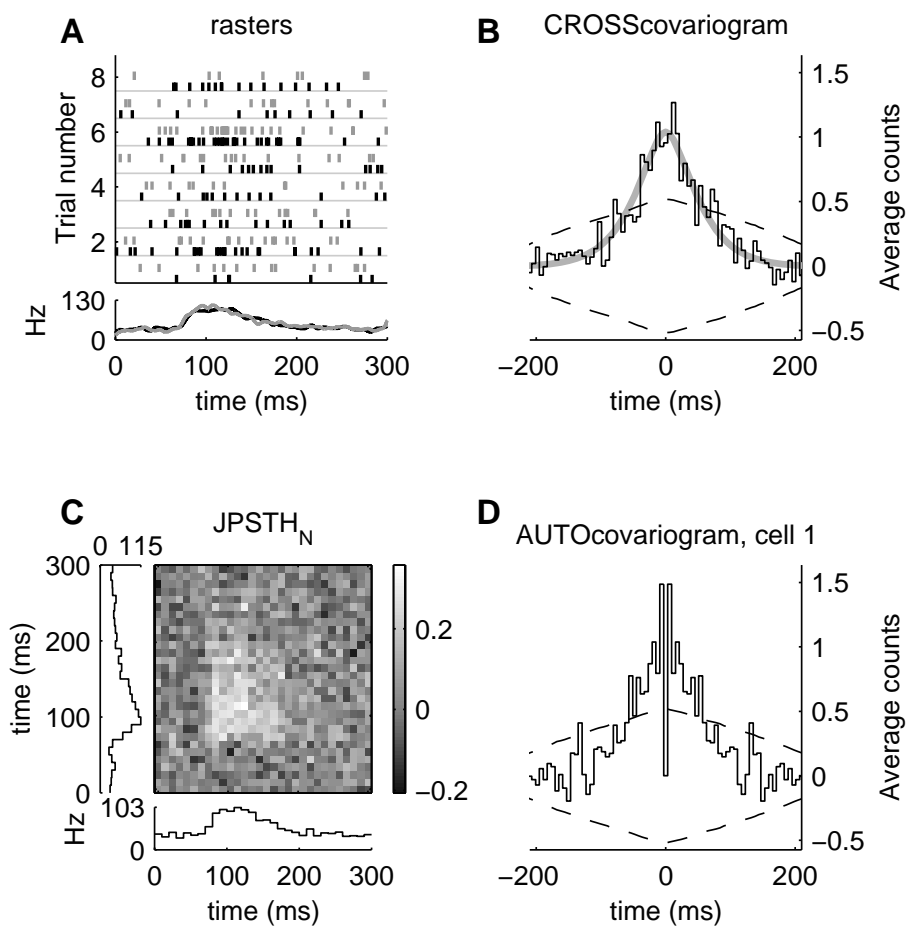

Figure 3: Excitability covariations. (A) Eight out of 200 rasters, made by simulating two independent Poisson cells with covarying gains $\zeta_{1}$ and $\zeta_{2}$ (see the text). Both gains were set to be equal to each other on each trial and were a random number drawn anew for each trial from a gaussian with mean 1 and standard deviation 1 (negative gains were set to zero). Below the rasters are the smoothed PSTHs of the two cells. $Z_{1}(t)$ and $Z_{2}(t)$ were both set to be, before multiplying by the gains, $(70 \mathrm{~Hz}) \cdot((t-70) / 30) \cdot \exp ((100-t) / 30)$ if $t>70$, zero otherwise, with $t$ in milliseconds. After multiplying by the gain, a constant rate (same for all trials) of $35 \mathrm{~Hz}$ was added to represent background firing. (B) Covariogram of the two cells. The thick gray line is the analytical expected value, computed from equation 3.3 with knowledge of the parameters used to generate the spike trains. Dashed lines are significance limits. Notice that the width of the peak is comparable to twice the width of the peak in the PSTHs. In the example here, the background firing rate is not negligible, so the covariogram does not quite follow the shape of the shuffle corrector (which is not shown), but follows the shape of $Z_{1} \odot Z_{2}$, the "stimulus-induced" parts of the PSTHs. (C) Normalized JPSTH of the two cells, flanked by their PSTHs. (D) Autocovariogram of one of the cells; the other is similar. The central bin $(\tau=0)$ has been set to zero for display purposes. 
Thus $\sum_{\tau} R(\tau)=\left\langle n_{1}^{r} n_{2}^{r}\right\rangle$. Similarly, $\sum_{\tau} K(\tau)=\left\langle n_{1}^{r}\right\rangle\left\langle n_{2}^{r}\right\rangle$. Hence

$$
\sum_{\tau} V(\tau)=\left\langle n_{1}^{r} n_{2}^{r}\right\rangle-\left\langle n_{1}^{r}\right\rangle\left\langle n_{2}^{r}\right\rangle=\operatorname{cov}\left(n_{1}, n_{2}\right)
$$

3.3 Spike Timing Covariations. Figure 4 shows a numerical experiment illustrating spike timing covariations. There are three major points in comparison to latency and excitability covariations. First, for the simple Poisson-like processes used here, where there was no burstiness and the spike timing interaction was between individual spikes of the two cells, the autocovariograms are flat and not at all similar to the cross-covariogram of the two cells. This is in contrast to the latency or excitability covariations cases and allows using the autocovariograms as a first test to distinguish spike timing from latency or excitability covariations. Second, although latency and excitability covariations involve coordination between as little as a single parameter of the two cells on each trial (overall latency in one case, gain in the other), spike timing covariations will typically involve coordination between many parameters on each trial (many individual spike times). Finally, given arbitrary network connectivities, spike timing covariogram shapes are much more arbitrary than latency or excitability covariogram shapes. Although the latter are tied to the shapes of the PSTHs, the former are not.

\section{Discussion}

Peaks in spike train covariograms are typically interpreted as evidence of spike timing synchronization, but other ways to depart from independence can generate covariogram peaks very similar to spike synchronization peaks. Two such departures have been described here: covariations in the latency of response and covariations in the excitability of response. Both are likely to be found in biological systems. This raises the possibility of covariograms that admit multiple, extremely different interpretations-an interpretation problem that must be solved. The first step in solving it is to be aware of under what conditions interpretational ambiguity may arise (and, concomitantly, when it can be ruled out). Contributing to this understanding has been the main objective of this article. The second step is to resolve the ambiguity when it is present; some quantitative methods for doing so are proposed in the companion article in this issue (see also Friston, 1995, and Vaadia et al., 1995). That excitability covariations could generate a peak in a JPSTH was a possibility raised by Aertsen et al. (1989), but they did not study the shape or magnitude of such a peak. Friston (1995; see also Vaadia et al., 1995) has described excitability covariations in more detail; similarities and differences between Friston's work and that presented here are discussed in the companion article in this issue. 

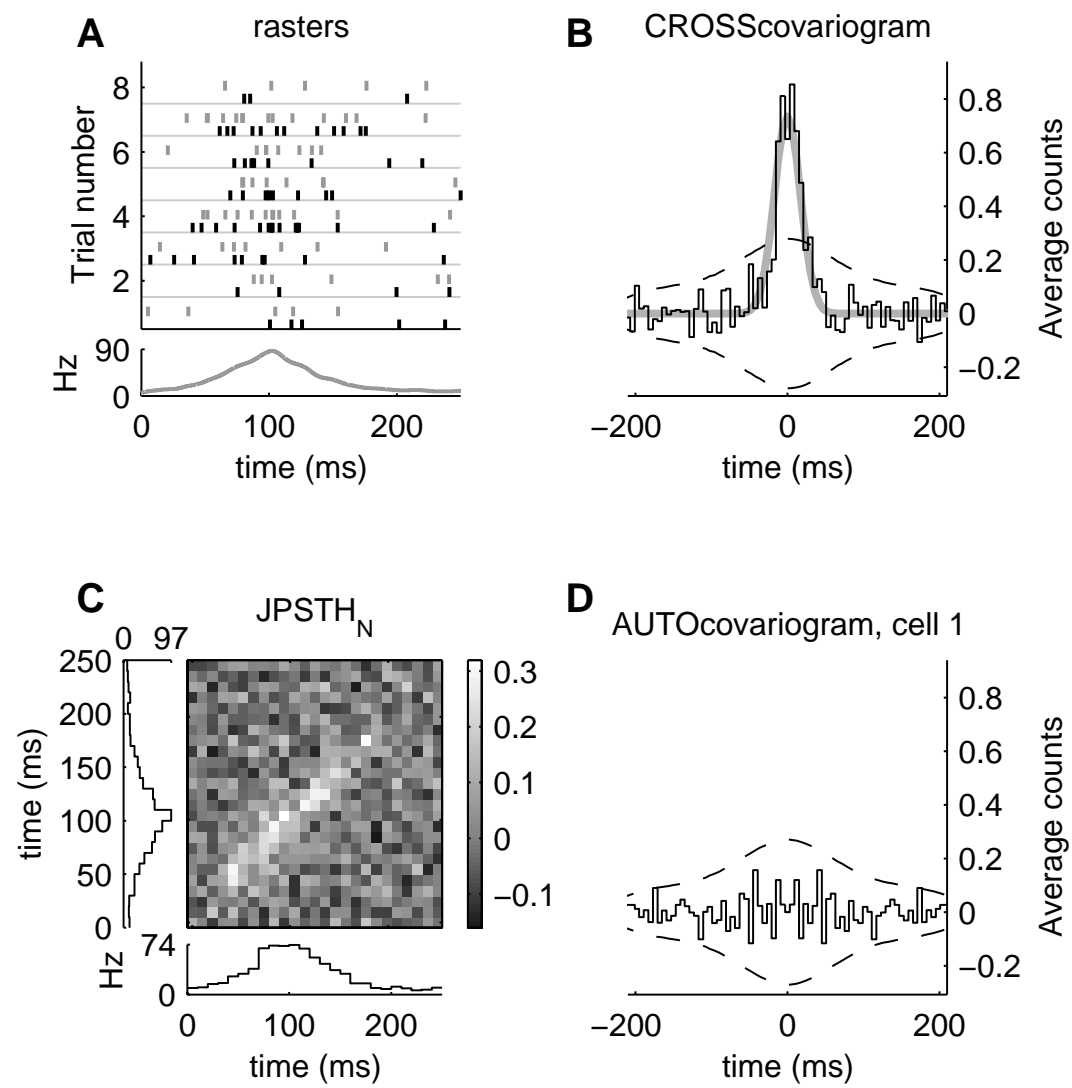

Figure 4: Spike timing covariations. (A) Eight out of 200 rasters used to illustrate spike timing covariations. Below the rasters are the smoothed PSTHs of the two cells. On each trial, the spike trains were made by first generating a single Poisson spike train, time-jittering the spikes of this twice, and then assigning the result of the first jittering to cell 1 and the result of the second jittering to cell 2. Additional spikes at a rate of $10 \mathrm{~Hz}$ were then added independently to each cell to represent background, uncorrelated, firing. The original spike train on each trial had firing rate $(70 \mathrm{~Hz}) \exp \left(-(t-100)^{2} /\left(2 \cdot 30^{2}\right)\right)$, with $t$ in milliseconds. Jittering was done by adding a random amount of time to each spike, drawn from a gaussian with mean 0 and standard deviation $12 \mathrm{~ms}$. (B) Covariogram of the two cells. The thick gray line is the analytical expected value of the covariogram, computed with knowledge of the parameters and procedures used to generate the spike trains. (C) Normalized JPSTH of the two cells, flanked by their PSTHs. (D) Autocovariogram of one of the cells; the other is similar. The central bin $(\tau=0)$ has been set to zero for display purposes. Unlike the latency and excitability cases in Figures 2 and 3, the autocovariogram does not resemble the cross-covariogram. 
Three rules of thumb, for being alert to whether latency or excitability covariations could be present in a covariogram, may be gleaned from the examples and results of section 3:

Rule of thumb 1: Covariogram peak widths due to latency and excitability covariations are of the same order of magnitude as PSTH peak widths. This is due to the fact that excitability peaks are directly linked to terms containing stimulus-locked components ${ }^{5}$ (in addition to background firing-rate terms as wide as the entire trial itself-see equation 3.3). Latency peaks depend on $R$, the raw correlogram peak width, which in turn depends on the characteristic width of the cells' responses (see Figure 2). Ken Britten (personal communication) has suggested estimating the characteristic width of the cells' responses from the autocovariograms instead of the PSTHs. This leads to rule of thumb 2:

Rule of thumb 2: Latency and excitability covariations generate autocovariogram peaks that are similar to the cross-covariogram peaks. While spike timing covariations may exist without affecting the cells' autocovariograms, latency or excitability covariations add a contribution to the autocovariogram that is similar to their contribution to the cross-covariogram. This was shown here with Poisson-like, nonbursty model cells. The statement remains true if both cells are equally bursty. But if the cells are not symmetric (e.g., one is bursty but the other is not), the comparison between auto- and cross-covariograms will no longer be straightforward.

Rule of thumb 3: The integral (i.e., sum) of a covariogram is directly proportional to the covariation in the mean firing rates of the neurons (see equation 3.6). Since the integral can be quickly estimated by eye, this measure should be in every covariogram-using neurophysiologist's breast pocket. ${ }^{6}$ Large, positive covariogram integrals indicate that the data were collected from trials with large, positive covariations in their firing rates (implying the presence of an excitability covariations component), and suggest important changes of state during the experiment. Examples of covariograms with large, positive integrals are common in the literature (Kruger \& Aiple, 1988; Alloway, Johnson, \& Wallace, 1993; Hata, Tsumoto, Sato, Hagihara, \& Tamura, 1993; Ghose, Ohzawa, \& Freeman, 1994; Sillito, Jones, Gerstein, \&

\footnotetext{
5 If the variations in the gain factors balance out-that is, if $\left\langle\zeta^{r}\right\rangle=0$ - the PSTHs may be flat even in the presence of excitability covariations (Friston, 1995).

6 The integral of the covariogram is exactly proportional to the covariation in mean firing rates when $S_{1}^{r}(t)$ and $S_{2}^{r}(t)$ are defined to be zero for times outside trial $r$ (see section 2 ), for the purpose of computing the covariogram. If this is not done, the integral will include a term describing covariations in mean firing rates for times surrounding the trials. But even in this case, positive integrals should prompt investigators to look at covariations in mean rates.
} 
West, 1994; Nowak, Munk, Nelson, James, \& Bullier, 1995; Munk, Nowak, Nelson, \& Bullier, 1995). Note that spike timing covariations, as illustrated in Figure 4, can also generate positive covariogram integrals (common input can lead to spike timing coincidences, but also to covariations in the number of spikes fired). However, in the spike timing case, the integral, if positive, will often be small, since the width of the peak can be very thin and unrelated to the width of the PSTH. Thus, the most telltale situation occurs when the integral is positive and the correlogram peak width is of the same order of magnitude as the PSTH peak widths. A straightforward method to determine whether the PSTHs can match the correlogram peak in this sense is presented in the companion article in this issue. (On the other hand, for examples of positive covariogram integrals clearly not caused by excitability covariations, see Tso, Gilbert, \& Wiesel, 1986.)

If any of the three rules of thumb suggests there could be important latency or excitability contributions to a covariogram, care should be taken before concluding that observed covariogram peaks are due to spike synchronization.

\section{Conclusion}

The three types of covariations that have been examined here are neither exhaustive nor exclusive. Other types of departure from independence also exist. Spike timing covariations may coexist with latency or excitability covariations, or both, and latency and excitability covariations, in particular, may commonly exist in a paired manner. John Hopfield (personal communication) has suggested that covariations in resting membrane potential could lead to paired covariations in both latency and excitability, since depolarized resting potentials would lead to both high excitabilities and short latencies, while hyperpolarized resting potentials would lead to both low excitabilities and long latencies. Such changes in resting potentials might be induced by variable ongoing activity in the network that the neurons are part of (see Arieli, Sterkin, Grinvald, \& Aertsen, 1996).

All covariations were illustrated here using stochastic processes that were constant over all the trials of each simulated experiment. Differences between trials were simply different instantiations of the same stochastic process. Thus, there is no sense in which the process generating the spike trains for Figure 1A (spike timing) was any more, or less, stationary than those of Figure 1B or Figure 1C (latency and excitability). Nevertheless, in biological systems, variations in latency or excitability would most likely be due to slow changes of state, which are indeed nonstationarities. When Aertsen et al. (1989) mentioned interpretation problems associated with excitability covariations, they phrased them as due to nonstationarities.

As an anonymous reviewer pointed out, the interpretation problems discussed in this article may be seen as a special case of a more general problem: that of taking the mean of a distribution as representative of all the points 
of the distribution. Only when the standard deviation of a distribution is much smaller than its mean ${ }^{7}$ can the latter be meaningfully thought of as representative of the entire distribution; in biological systems, distributions are often broad, and this condition is often not met. For example, the PSTH is defined as the average response over a set of trials, but if there are large variations in latency or excitability, it is clearly not representative of each individual trial. Similarly, the covariogram is defined averaged over a set of trials and should not necessarily be taken as representative of interactions occurring on each individual trial. Investigators must interpret means with care.

\section{Acknowledgments}

I am grateful to John Hopfield and members of his group, Sanjoy Mahajan, Sam Roweis, and Erik Winfree, for discussion and critical readings of the manuscript for this article. I also thank John Hopfield for support. I thank George Gerstein, Kyle Kirkland, and Adam Sillito for discussion, and the anonymous reviewers for helpful comments. This work was supported by a Fulbright/CONACYT graduate fellowship and by NSF Cooperative Agreement EEC-9402726.

All simulations and analyses were done in Matlab 5 (Mathworks Inc., Natick, MA). The code for all of these, including in particular the code to reproduce each of the figures, can be found at http://www.cns.caltech.edu / $\sim$ carlos/correlations.html.

\section{References}

Aertsen, A. M. H. J. Gerstein, G. L., Habib, M. K., \& Palm, G. (1989). Dynamics of neuronal firing correlation-modulation of effective connectivity. J. Neurophysiol., 61(5), 900-917.

Alloway, K. D., Johnson, M. J., \& Wallace, M. B. (1993). Thalamocortical interactions in the somatosensory system-interpretations of latency and crosscorrelation analyses. J. Neurophysiol., 70(3), 892-908.

Arieli, A., Sterkin, A., Grinvald, A., \& Aertsen, A. (1996). Dynamics of ongoing activity-explanation of the large variability in evoked cortical responses. Science, 273, 1868-1871.

Brody, C. D. (1997a). Analysis and modeling of spike train correlations in the lateral geniculate nucleus. Unpublished doctoral dissertation, California Institute of Technology, Available at http://www.cns.caltech.edu/ $\sim$ carlos/thesis.

Brody, C. D. (1997b). Latency, excitability, and spike timing correlations. Society for Neuroscience Abstracts, 23, 14.

\footnotetext{
${ }^{7}$ For multivariate distributions, the square root of all eigenvalues of the covariance matrix must be much smaller than the magnitude of the mean.
} 
Carandini, M., \& Ferster, D. (1997). A tonic hyperpolarization underlying contrast adaptation in cat visual-cortex. Science, 276, 949-952.

Friston, K. J. (1995). Neuronal transients. Proceedings of the Royal Soc. of London Series B Biological Sciences, 261, 401-405.

Ghose, G. M., Ohzawa, I., \& Freeman, R. D. Receptive-field maps of correlated discharge between pairs of neurons in the cat's visual-cortex. J. Neurophysiol., 71(1), 330-346.

Hata, Y., Tsumoto, T., Sato, H., Hagihara, K., \& Tamura, H. (1993). Development of local horizontal interactions in cat visual-cortex studied by crosscorrelation analysis. J. Neurophysiol., 69(1), 40-56.

Kruger, J. J., \& Aiple, F. (1988). Multimicroelectrode investigation of monkey striate cortex-spike train correlations in the infragranular layers. J. Neurophysiol., 60(2), 798-828.

Munk, M. H. J., Nowak, L. G., Nelson, J. I., \& Bullier, J. (1995). Structural basis of cortical synchronization. Effects of cortical-lesions. J. Neurophysiol., 74(6), 2401-2414.

Nowak, L. G. , Munk, M. H. J., Nelson, J. I., James, A. C., \& Bullier, J. (1995). Structural basis of cortical synchronization. 3. Types of interhemispheric coupling. J. Neurophysiol., 74(6), 2379-2400.

Palm, G., Aertsen, A. M. H. J., \& Gerstein, G. L. (1988). On the significance of correlations among neuronal spike trains. Biological Cybernetics, 59(1), 1-11.

Perkel, D. H., Gerstein, G. L., \& Moore, G. P. (1967). Neuronal spike trains and stochastic point processes. II. Simultaneous spike trains. Biophysics Journal, 7, 419-440.

Sillito, A. M., Jones, H. E., Gerstein, G. L., \& West, D. C. (1994). Feature-linked synchronization of thalamic relay cell firing induced by feedback from the visual-cortex. Nature, 369(6480), 479-482.

Tso, D. Y., Gilbert, C. D., \& Wiesel, T. N. (1986). Relationships between horizontal interactions and functional architecture in cat striate cortex as revealed by cross-correlation analysis. J. Neurosci., 6(4), 1160-1170.

Vaadia, E., Aertsen, A., \& Nelken, I. (1995). "Dynamics of neornal interactions" cannot be explained by "neuronal transients". Proceedings of the Royal Soc. of London Series B Biological Sciences, 261, 407-410. 